\title{
DESING ON WIRELESS INTELLIGENT SENEOR NETWORK ON CLOUD COMPUTING SYSTEM FOR SMART HOME
}

\author{
Tsung-Han Tsai, Chih-Chi Huang, and Chih-Hao Chang \\ Department of Electrical Engineering, National Central University, Taiwan
}

\begin{abstract}
Sensors on (or attached to) mobile phones can enable attractive sensing applications in different domains such as environmental monitoring, social networking, healthcare, etc. In this paper we propose a cloud computing system dedicated on smart home applications. We design the proposed wireless vision sensor network (WVSN) with its algorithm and hardware implementation. In WVSN, The partial-vision camera strategy is applied to allocate the computation task between the sensor node and the central server. Then we propose a high performance segmentation algorithm. Meanwhile, an efficient binary data compression method is proposed to cope with the result on labeling information. The proposed algorithm can provide high precision rate for the smart home applications such as the gesture recognition and humanoid tracking. To realize the physical system, we implement it on the embedded platform and the central server with their transmission work.
\end{abstract}

\section{INTRODUCTION}

Mobile devices (smart phones, tablets, laptops, embedded boards, robots) can serve as terminals for cloud computing services over intelligent network. Mobile cloud has emerged as a new cloud computing platform that 'puts cloud into a pocket'. Most of current devices are equipped with a rich set of embedded sensors such as camera, GPS, WiFi/3G/4G radios, accelerometer, digital compass, gyroscope, microphone and so on. These sensors can enable attractive sensing applications in various domains such as environmental monitoring, social networking, healthcare, transportation, safety, etc.

Smart home is a trend on modern human life. It is dominated by different kinds of sensors over the wireless network. The smart appliance is controlled by the central processor to achieve electrical automation features such as: lights, TV, air conditioning, security systems, etc. With a success on smart home, many functions can be explored, especially at home care, convenient and energy-saving environmental protection respectively. The concept of smart home with the versatile human-style applications is shown in Fig. 1.

To realize a smart home as a cloud computing system with the relative applications, sensor network technique is deployed as a service platform. Most deployed wireless sensor networks measure scalar physical phenomena such as temperature, pressure, humidity, or location of Dhinaharan Nagamalai et al. (Eds) : ACITY, WiMoN, CSIA, AIAA, DPPR, NECO, InWeS - 2014 pp. 103-111, 2014. (C) CS \& IT-CSCP 2014

DOI : $10.5121 /$ csit.2014.4512 
objects. More recently, intelligent monitoring system began to have a new breakthrough in image recognition. The original image by the person viewing the monitor mode is gradually developed into automatically monitored by a computer. Then the system can directly give a warning to person. Some applications are mostly eager to this solution, including: vehicle license plate recognition, falls among the elderly, face department identification, fire identification, etc. With this technology, many semantic applications on a smart home are realized.

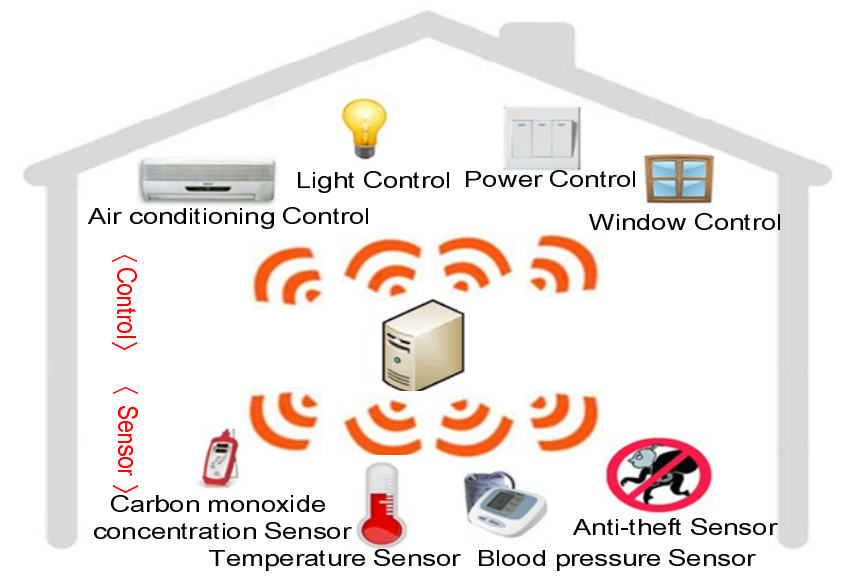

Fig. 1. Schemes of smart home.

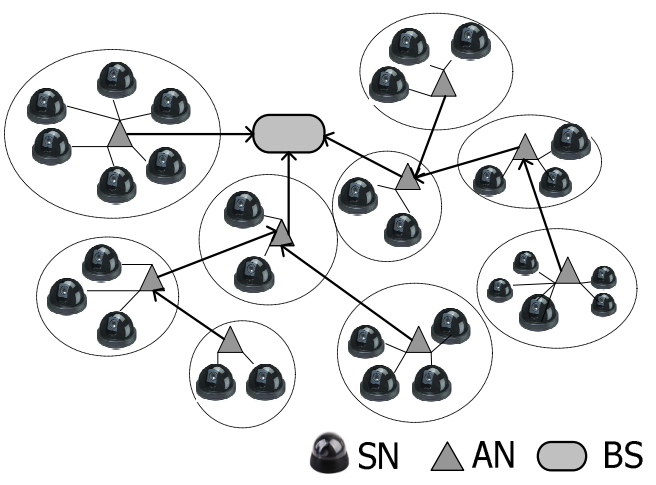

Fig. 2. Topology of WVSN.

In this paper, we design a wireless intelligent sensor network on cloud computing system for smart home applications. First, the Wireless Vision Sensor Network (WVSN) platform is investigated and the design issue for smart home is concerned. Here we propose a strategy which is more dedicated to smart home scenario. Second, we propose an object segmentation algorithm with a high performance and low complexity considerations. Third, to cope with the limited bandwidth on transmission, the foreground data is still needed to compress into a smaller amount of data. This paper is organized as follows. Section II briefly reviews the relevant literature in the aspect of smart home and the design challenges. Section III explores the whole algorithm in detail. Section IV presents the experimental results. Finally, Section V draws a conclusion. 


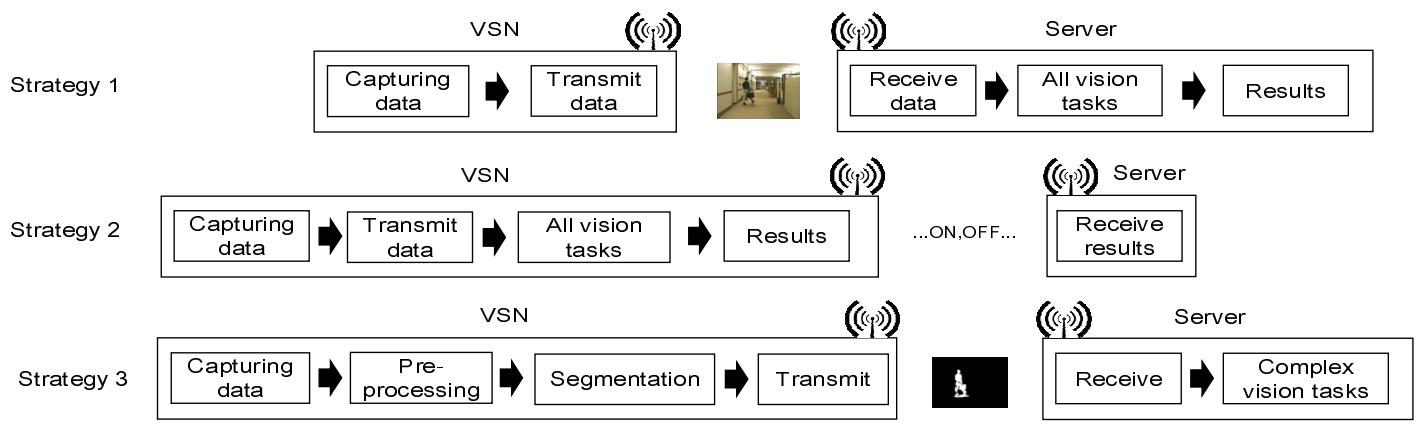

Fig. 3. Three strategies on WVSN architecture

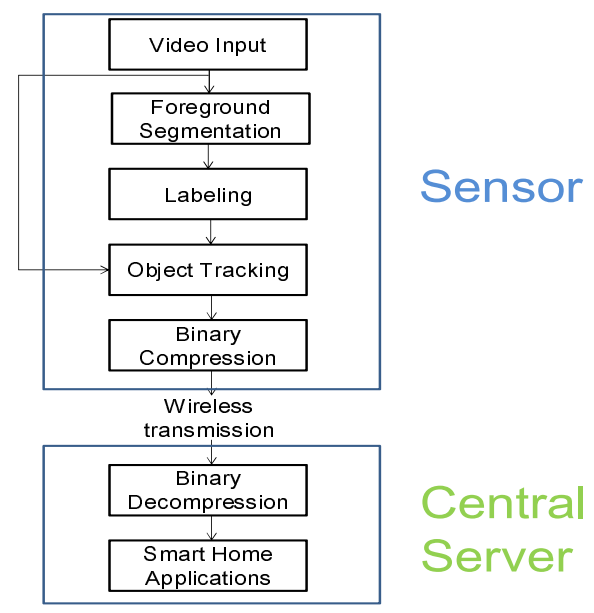

Fig. 4. Flowchart of the whole system on smart home.

\section{BACKGROUND ON RELATED WORK}

This paper targets on the smart home scene captured by the wireless video sensor nodes. To accomplish it, the concept on WVSN is first constructed. Then the related techniques on vision processing with their challenges are discussed later.

\section{A. WVSN}

Video data on wireless sensor network is accomplished as Wireless Vision Sensor Network [1]. It is constructed based on the sensor network, as shown in Fig. 2. In WVSN, each sensor node is tasked to capture video data and is capable of performing specific content analysis tasks to extract information from the video. The captured video and the extracted information are delivered to an aggregation node (AN). The role of the AN is to process the collected data and deliver important information to the base station (BS) [1]. WVSN has been envisioned for a wide range of important applications, including security monitoring, environment tracking, and the assisted living [2]. A design with distributed interactive video arrays for situation awareness of traffic and incident monitor was presented in [3]. A multicamera tracking adjacent cameras was proposed in [4]. 
Currently a well-established VSN, Zigbee, is widely applied. Zigbee had flexible network structure; it could support the topology of star shape, tree shape and mesh shape [5]. Mesh structure maintains ad-hot feature on VSN however it is not easily realized on real environment. Star and binary tree structure contains less variation but the robust is controllable.

\section{B. Design Challenges}

WVSN will be enabled by the convergence of communication and computation with signal processing and several branches of control theory and embedded computing. To realize the WVSN, it often exposes to a number of challenges. Generally, researchers employ three strategies for WVSN implementation [4]-[7], as shown in Fig. 3.

In the first strategy, no local processing is performed on the VSN and raw data is directly transmitted to the server for vision processing. However, this strategy consumes large transmission time because the large amount of data is communicated [8]. Moreover, in a house environment, privacy should be the most concerned issue. The video data is transmitted on air and thus this could not be accepted in many personal and private environments.

In the second strategy, all vision tasks are performed on the VSN and only the final features are transmitted to the server for analysis [4]. The advantage is that no visible data is transmitted on air and consequently the privacy is fully reserved. This strategy forces the VSN to consume large processing effort on the currently available software platforms and has high design complexity on the hardware platforms.

Referring to the third strategy, it moves the complex tasks i.e., labeling, feature extraction and classification, to a server. This strategy will reduce both the processing consumption and the design complexity because the complex tasks are moved to a generalized platform on the server.

\section{PROPOSED AlgorithM}

In this paper, we adopt the third strategy to realize the WVSN on a smart home. We propose a high performance object segmentation algorithm dedicated to this scenario. As shown in Fig. 4, the proposed system can be divided into foreground segmentation, object labeling, object tracking, binary data compression, and the smart home applications. Details are shown as follows.

\section{A. Foreground Segmentation}

The foreground detection is meant to separate the interest objects in video. It is always the most crucial and important task in an object segmentation flow. The first step is the background modeling where we consider the rate control issue. The second step is the background subtraction and the third step is the area filter. Background subtraction method is a comprehensive concept and successfully utilized on background modeling and moving-object detection. We modified the algorithm on [9] to perform the background modeling with a more rapid convergence rate. The proposed algorithm utilizes Gaussian mixture models (GMM) and adaptive mixture learning. By this technology, each pixel is framed as a mixture of Gaussians. Then the on-line approximation is used to improve it. Here we evaluate these Gaussian distributions to judge the most likely result. 


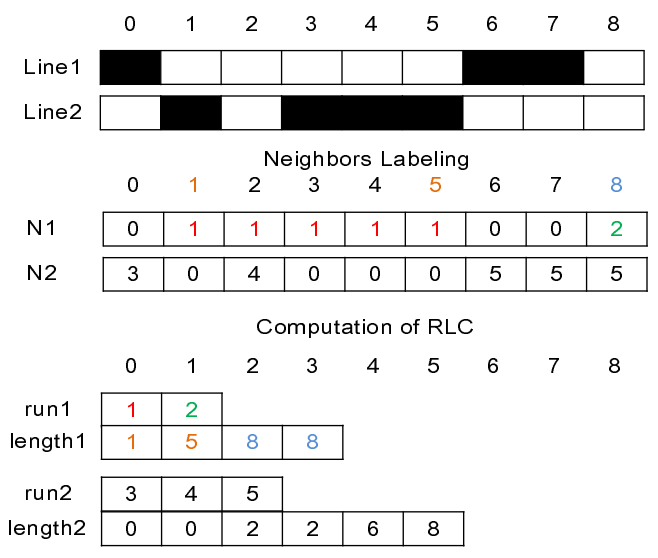

Fig. 5. Neighbors labeling and RLC.

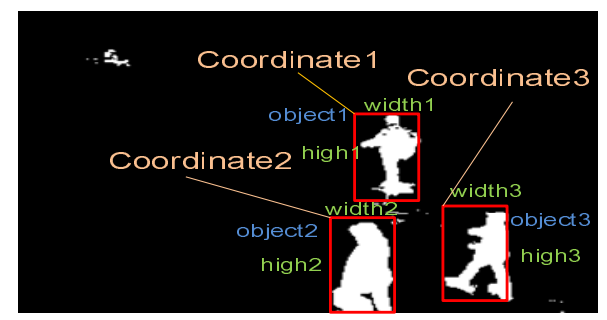

Fig. 6. Object information.

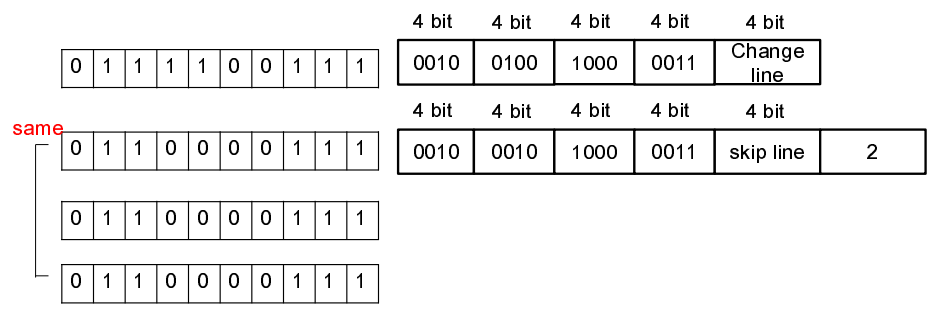

Fig. 7. RLC with skip-line algorithm.

\section{B. Object Labeling}

After we have the binary foreground mask, we have to label the connect pixel. We need the object labeling algorithm with fast and low complexity issue. We use the approach where it is a combination of run-length encoding (RLC) and object labeling of algorithms. Fig. 5 shows the neighbors labeling and calculation on RLC. Our approach will label line by line. Only the foreground data, representing as white, is needed with labeling. As shown in Fig. 5, in the first line N1, the data on position 1-5 are connected together. They will be given as the same number, where 1 (marked as red) is the run of position 1-5, and 2 (marked as green) is run of position 8 . The second line N2 is performed with the same procedure. Then we derive a run-length representation for $\mathrm{N} 1$ (run1, length1) and N2 (run2, length2) respectively. 


\begin{tabular}{|c|c|c|c|}
\hline & Original & Truth map & Proposed \\
\hline \multirow{2}{*}{ A1_88 } & & & \\
\hline \multirow{2}{*}{\multicolumn{4}{|c|}{ A1_165 }} \\
\hline & & & \\
\hline \multirow{2}{*}{\multicolumn{4}{|c|}{ A2_109 }} \\
\hline & & & \\
\hline \multicolumn{4}{|l|}{ A2_825 } \\
\hline \multicolumn{4}{|l|}{ A3_498 } \\
\hline & & $k$ & $\dot{\Lambda}$ \\
\hline A3_1378 & & & \\
\hline & & $\lambda$ & 3 \\
\hline
\end{tabular}

Fig. 8. Result of foreground segmentation

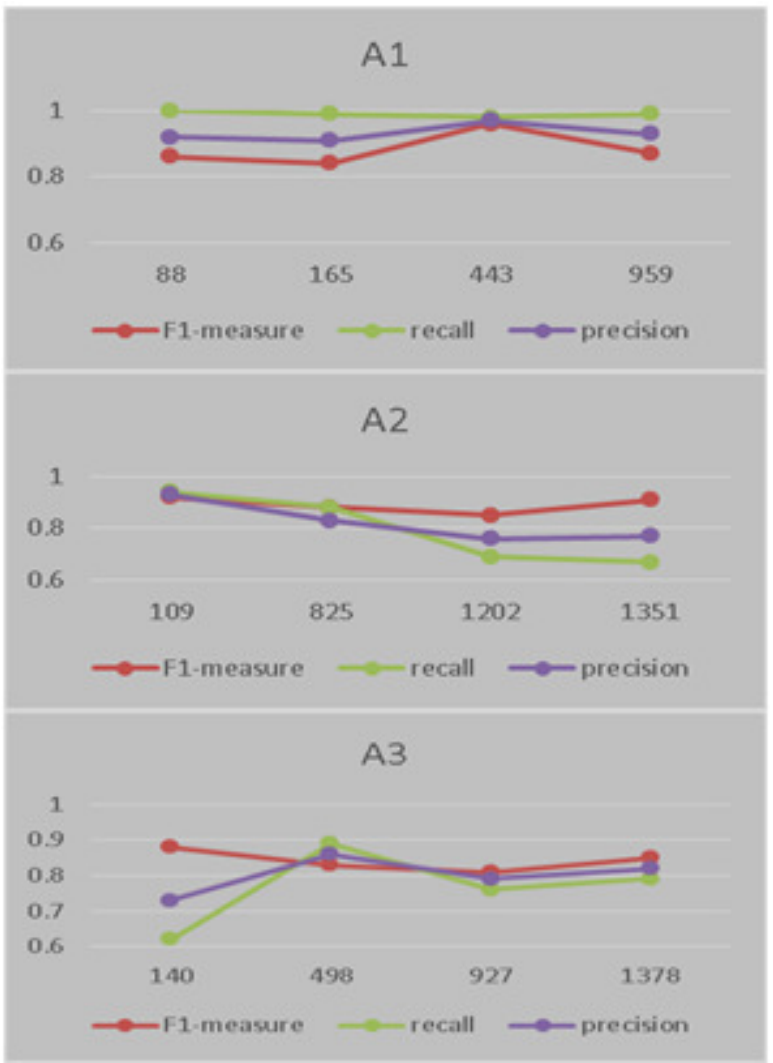

Fig. 9. The evaluation of the foreground segmentation. 


\section{Object Tracking}

We use the color feature to establish a color distribution model. Then we use a Kernel-based algorithm. If the model already exists, we can just track it. If this model does not exist, we create a new model. When an object moves, we can directly use the object labeling information to find the object. When the object is stationary, we can use the mean-shift algorithm to find the candidate model. Kernel-based tracking must meet a number of conditions. Meanwhile, the object cannot move faster than a certain speed and the kernel of the object cannot change too much.

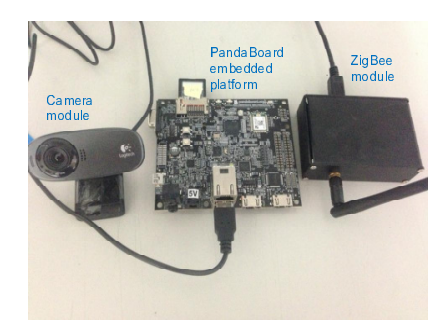

Fig. 10. View of the embedded system

\section{Binary Data Compression}

Now we have successfully tracked all objects, and each object is given with a label and recorded with the coordinates. This information facilitates our binary data compression. Taking into account the issue of privacy and data size, we will send the foreground object mask and size also the coordinate information, as shown in Fig. 6. Since we have tracked each object separately, we can use this information to calculate the size of each object.

The main compression technique is run-length coding with an additional concept on skip-line method. As a foreground image, two concessive rows usually have high degree of similarity. When many lines of data are the same, if we use this part of a symbol to represent. As shown Fig. 7 , since the data in the first and second line is different, the data on line 2 is kept completely. From the second line to the forth line, all the data are identical. In that case we just add a skipline symbol. The value of this symbol is 2 representing that there are two more identical rows existed.

\section{EXPERIMENTAL RESULTS}

In order to verify the results of our algorithm, we will simulate the individual algorithm with the experimental result. Some evaluation and comparison are provided.

We dedicate many experiments on foreground segmentation. Obviously foreground segmentation will directly affect the accuracy of performance. If the some unnecessary portions are included, the system will waste time on scanning and judgment. Also if the cut is less the truth case, it will lead to the failure or error on judgment. We analyze the results and draw their own truth map. Fig. 8 shows the truth map and the prospect the proposed algorithm cut out. By this subjective view, the similarity between them is very high. To measure the accuracy, the recall rate, precision 
and F1-measure are the most comprehensive assessments. In our three test sequences, F1measure can reach above 0.8. The evaluation is shown in Fig. 9.

Referring to the compression rate analysis, because our binary data compression method is a lossless technique, no quality loss is existed between the source and decoded result. The compression ratio of our method varies from 20 to 500 in some frame. In average 50 times of compression ratio is achieved.

The coordinator is as our central server. All end devices are the sensors which can only return data to a central server.

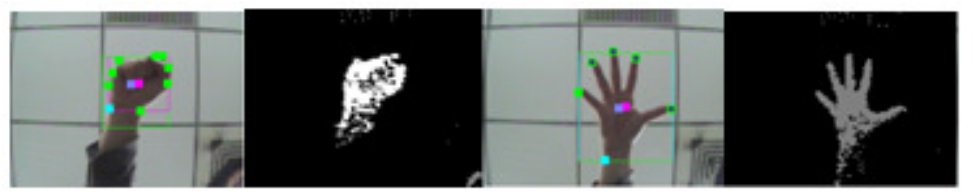

(a) Gesture A

(b) Gesture B

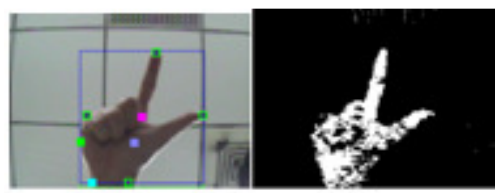

(b) Gesture C

Fig. 11. Hand gesture recognition result.

Then the server can determine which end device is now transmitting the data. In the VSN, a camera is plug at an embedded system board. Here we use the PandaBoard embedded platforms with camera and Zigbee modules. Fig. 10 shows the view of the embedded system. This board is based on ARM Cortex-A9 dual-core processor and has 1GB memory for usage. We use USB cameras and USB Zigbee modules. Several gestures are identified by the server. Fig. 11 shows the different gesture recognition results.

\section{CONCLUSION}

In this paper, we realize a smart home based on the implementation on WVSN as a cloud computing system. Due to different design philosophies on WVSN, this paper applies a low complexity approach which concerns the characteristics on the VSN and the server. For the algorithm level, a complete system with efficient vision-based tasks is provided. Versatile test sequences are simulated to prove the feasibility of the propose algorithm. Furthermore, a physical system of WVSN is constructed and some intelligent applications are realized.

\section{REFERENCES}

[1] F. Akyildiz, T. Melodia, and K. R. Chowdhury, "A survey on wireless multimedia sensor networks," Elsevier Comput. Netw., vol. 51, pp. 921-60, Mar. 2007.

[2] M. Valera and S. Velastin, "Intelligent distributed surveillance systems: A review," IEEE Proc. Vis. Image, Signal Process., vol. 152, no. 2, pp. 192-204, Apr. 2005. 
[3] M. M. Trivedi, T. L.Gandhi, and K. S. Huang, "Distributed interactive video arrays for event capture and enhanced situational awareness," IEEE Intell. Syst., vol. 20, no. 5, pp. 58-66, Oct. 2005.

[4] M. Quaritsch, M. Kreuzthaler, B. Rinner, H. Bischof, and B. Strobl, "Autonomous multicamera tracking on embedded smart cameras," in EURASIP J. Embed. Syst., 2007.

[5] F. Akyildiz, T. Melodia, and K. R. Chowdhury, "A survey on wireless multimedia sensor networks," Elsevier Comput. Netw., vol. 51, pp. 921-60, Mar. 2007.

[6] Imran, M.; Ahmad, N.; Khursheed, K.; Waheed, M.A.; Lawal, N.; O'Nils, M., "Implementation of Wireless Vision Sensor Node With a Lightweight Bi-Level Video Coding," Emerging and Selected Topics in Circuits and Systems, IEEE Journal on , vol.3, no.2, pp.198,209, June 2013.

[7] M. Imran, K. Khursheed, M. O'Nils, and N. Lawal, "Exploration of target architecture for a wireless camera based sensor node," in IEEE Norchip Conf., Nov. 2010, pp. 1-4.

[8] S. Soro and W. Heinzelman, "A survey of visual sensor networks," Adv. Multimedia, pp. 1-22, May 2009.

[9] D.-S. Lee, "Effective Gaussian Mixture Learning for Video Background Subtraction," IEEE Transactions on Pattern Analysis and Maching Intelligence, vol. 27, no. 5, MAY 2005. 